\title{
THE TEICHMÜLLER SPACES ARE DISTINCT
}

\author{
DAVID B. PATTERSON
}

\begin{abstract}
The Teichmüller space $T(g, n)$ of a compact Riemann surface of genus $g$ with $n$ punctures is a complex manifold of $\operatorname{dim}=3 g-3+n$. In any given dimension, there are a finite number of these Teichmüller spaces and it is natural to ask if all of these are distinct (up to biholomorphic equivalence). We have shown here that with the exception of two special cases in dimensions 1 and 3 all of these spaces are distinct, that is, $T(g, n)$ is not biholomorphically equivalent to $T\left(g^{\prime}, n^{\prime}\right)$ unless $g^{\prime}=g$ and $n^{\prime}=n$.
\end{abstract}

A surface of finite type $(g, n)$ is a compact Riemann surface of genus $g$ with $n$ distinct points (punctures) removed. For $3 g-3+n>0$, let $T(g, n)$ be the Teichmüller space of a surface of type $(g, n)$ and $\operatorname{Mod}(g, n)$ its modular group. For the definitions and properties of these spaces see Bers [1]. These spaces are all finite-dimensional complex manifolds and $\operatorname{dim} T(g, n)=3 g-3+n$. For suitable values of $g$ and $n$, two such spaces will have the same dimension and, a priori, could be the same, that is, biholomorphically equivalent. That this can, in fact, happen is shown by the following known cases:

1. $T(0,4) \cong T(1,1)$,

2. $T(0,6) \cong T(2,0)$.

THEOREM. Except for the two cases listed above, $T(g, n)$ is not biholomorphically equivalent to $T\left(g^{\prime}, n^{\prime}\right)$ unless $g=g^{\prime}$ and $n=n^{\prime}$.

$\operatorname{Mod}(g, n)$ is canonically isomorphic to the mapping class group of a surface $S$ of type $(g, n)$, that is, the group of all orientation preserving homeomorphisms of $S$ modulo those homotopic to the identity. $\operatorname{Mod}(g, n)$ acts as a group of analytic automorphisms of $T(g, n)$ and this action is effective if $3 g-3+n>1$ and $(g, n) \neq(2,0)$. By a recent result of Royden ([12], [13]), $\operatorname{Mod}(g, n)$ is, in fact, the full group of analytic automorphisms of $T(g, n)$ if $3 g-3+n>1$ and if $\operatorname{Mod}(2,0)$ is interpreted here as the effective group of automorphisms of $T(2,0)$. (Royden states the result explicitly only for the case of compact surfaces of genus $g \geqq 2$, but the

Received by the editors October 26, 1971.

AMS 1970 subject classifications. Primary 32G15.

Key words and phrases. Teichmüller space, modular group, mapping class group, Riemann surfaces.

c American Mathematical Society 1972 
argument carries through in the general case.) Thus it follows that if two Teichmüller spaces are the same, then their modular groups must be the same group of analytic automorphisms of this space.

An element $\gamma^{*}$ of finite order in $\operatorname{Mod}(g, n)$ is represented by a (conformal) automorphism $\gamma$ of a surface $S$ of type $(g, n)$ having this order. For compact surfaces, this was first proved algebraically by Nielsen ([9], [10]), other proofs have been given by Fenchel ([3], [4]) and Macbeath [7]. For a cyclic subgroup of prime-power order, this result is a direct consequence of $\mathrm{P}$. A. Smith's fixed point theorem [14] and the fact that $T(g, n)$ is homeomorphic to a finite-dimensional real Euclidean space. The analogous result for a finite cyclic and, more generally, a finite solvable subgroup of $M(g, n)$ follows by means of an inductive argument. See Kravetz [5, p. 27] for a sketch of these proofs.

If $\Gamma$ and $\Gamma^{*}$ denote the cyclic groups generated by $\gamma$ and $\gamma^{*}$ respectively and $\operatorname{Fix}(\Gamma)$ and $\operatorname{Fix}\left(\Gamma^{*}\right)$ denote their fixed point sets, then $\operatorname{Fix}\left(\Gamma^{*}\right)$ is an analytic submanifold of $T(g, n)$ which is isomorphic to the Teichmüller space of the quotient surface $(S-\operatorname{Fix}(\Gamma)) / \Gamma$ (see, for instance, Rauch [11]). We will use this to prove the following lemma. In what follows we will always assume that $3 g-3+n>1$ and $(g, n) \neq(2,0)$.

Lemma 1. The dimension of the fixed point set of any involution in $\operatorname{Mod}(g, n)$ is less than or equal to $2 g-1+[n / 2]$, where $[x]$ denotes the largest integer $\leqq x$. Morcover, $\operatorname{Mod}(g, n)$ aliays contains involutions $\gamma^{*}$ such that this dimension is precisely $2 g-1+[n / 2]$. This occurs if and only if $\gamma^{*}$ is represented by an involution $\gamma$ with $2 g+2$ fixed points which fixes no punctures if $n$ is even and which fixes exactly one puncture if $n$ is odd.

Proof. An involution $\because *$ in $\operatorname{Mod}(g, n)$ is represented by an involution $\gamma$ on a surface $S$ which fixes $m$ punctures and which has $k$ fixed points in all. By the Riemann-Hurwitz formula, $(S-\operatorname{Fix}(\Gamma)) / \Gamma$ is a surface of genus ${ }_{2}^{1} g+\frac{1}{2}-{ }_{4}^{1} k$ with $k+\frac{1}{2}(n-m)$ punctures. Thus the fixed point set of $\gamma^{*}$ had dimension ${ }_{2}^{3}(g-1)+{ }_{2}^{1} n+\frac{1}{4}(k-2 m)$. Since an involution can have at most $2 g+2$ fixed points and since $n-m$ must be even, the maximum possible dimension is obtained when $k=2 g+2$ and $m=0$ if $n$ is even and $m=1$ if $n$ is odd. The maximum value $2 g-1+[n / 2]$ is always obtained, since there are surfaces having an involution with $2 g+2$ fixed points, namely the "hyperelliptic" ones.

Now, the number $2 g-1+[n / 2]$ is defined intrinsically in terms of the space $T(g, n)$. Thus, if $T(g, n) \simeq T\left(g^{\prime} \cdot n^{\prime}\right)$, then we would have:

$$
3 g-3+n=3 g^{\prime}-3+n^{\prime}, \quad 2 g-1+[n / 2]=2 g^{\prime}-1+\left[n^{\prime} / 2\right] .
$$

If $n$ and $n^{\prime}$ are both even or both odd, these equations have only one solution $g=g^{\prime}$ and $n=n^{\prime}$. If one of these, say $n$, is even and the other, say 
$n^{\prime}$, is odd, then these equations have only one solution $g^{\prime}=g+1$ and $n^{\prime}=n-3$. Hence the only possible exceptions to the theorem occur in the latter case.

To distinguish between these spaces, we define a maximal involution to be an involution in $\operatorname{Mod}(g, n)$ whose fixed point set has dimension as large as possible, namely $2 g-1+[n / 2]$. If two such spaces were the same, then the centralizers of maximal involutions would be the same. According to Lemma 2 below, however, this is impossible for the pair of spaces $T(g, n)$ and $T(g+1, n-3)$. Hence the theorem follows once we establish:

Lemma 2. There is a maximal involution $\gamma^{*}$ in $\operatorname{Mod}(g, n)$ whose centralizer contains an involution distinct from $\gamma^{*}$ if and only if $n$ is even.

Proof. If $\beta^{*}$ is an involution in the centralizer of a maximal involution $\gamma^{*}$, then by an extension of Nielsen's theorem $\beta^{*}$ and $\gamma^{*}$ can be represented by a pair of commuting involutions $\beta$ and $\gamma$ on a surface. Since $\gamma^{*}$ is maximal, $\gamma$ has $2 g+2$ fixed points and the surface is "hyperelliptic" with $\gamma$ as its "hyperelliptic" involution. Moreover, if $n$ is odd, $\gamma$ must fix one of the punctures. Let $W$ be the set of fixed points of $\gamma$. These are the so-called Weierstrass points of $S$. Since $\beta$ commutes with $\gamma, \beta$ maps $W$ onto $W$. Moreover, since $\beta$ is an involution and $W$ contains an even number of points, $\beta$ must fix an even number of the points of $W$. Since $\beta$ also maps punctures to punctures, $\beta$ must fix the unique puncture which lies in $W$. Thus $\beta$ fixes one and, hence, at least two of the points of $W$. By a theorem of Lewittes [6, Theorem 7, p. 748], this can happen only if $\beta=\gamma$. On the other hand, if $n$ is even, it is easy to construct a surface with two such involutions $\beta$ and $\gamma$ with $\beta \neq i$. In fact, $\beta$ and $\gamma$ can be realized as a pair of symmetries in 3-space.

Finally, one suspects that even more may be true; namely $\operatorname{Mod}(g, n)$ is not isomorphic (as an abstract group) to $\operatorname{Mod}\left(g^{\prime}, n^{\prime}\right)$ in general. This would be a stronger result which would imply the same theorem, but which cannot be proven by the present method. In some cases this can be proven by finding an element of finite order in one group which does not occur in the other; however, this method will not suffice in general. One result along these lines is as follows. Birman [2] has shown that for $g \geqq 3$, $\operatorname{Mod}(g, 0)$ modulo its commutator subgroup is cyclic of order 1 or 2 . Using the presentation of $\operatorname{Mod}(0, n)$ given by Magnus [8], we find that $\operatorname{Mod}(0, n)$ modulo its commutator subgroup is cyclic of order $2(n-1)$ if $n$ is even and $n-1$ if $n$ is odd. Thus $\operatorname{Mod}(g .0)$ is not isomorphic to $\operatorname{Mod}(0, n)$ if $g \geqq 3$.

ACKNOWLEDGMent. The author wishes to thank Professor Lipman Bers for suggesting this problem and for indicating that Royden's theorem might be used to solve it. 


\section{REFERENCES}

1. L. Bers, Uniformation, moduli, and Kleinian groups, Bull. London Math. Soc. (to appear).

2. J. Birman, Abelian quotients of the mapping class group of a 2-manifold, Bull. Amer. Math. Soc. 76 (1970), 147-150. MR 40 \#2846.

3. W. Fenchel, Estensioni di gruppi discontinui e transformazioni periodiche delle superficie, Atti Accad. Naz. Lincei Rend. Cl. Sci. Fis. Mat. Nat. (8) 5 (1948), 326-329. MR 10, 558.

4. - Bemaerkinger om endelige grupper af afbildningsklasser, Mat. Tidsskr. B. 1959, 90-95.

5. S. Kravetz, On the geometry of Teichmüller spaces and the structure of their modular groups, Ann. Acad. Sci. Fenn. Ser. AI No. 278 (1959), 35 pp. MR 26 \#6402.

6. J. Lewittes, Automorphisms of compact Riemann surfaces, Amer. J. Math. 85 (1963), 734-752. MR 28 \#4102.

7. A. M. Macbeath, On a theorem by J. Nielsen, Quart. J. Math. Oxford Ser. (2) 13 (1962), 235-236. MR 25 \#4094.

8. W. Magnus, Über Automorphismen von Fundamentalgruppen berandeter Flächen, Math. Ann. 109 (1934), 617-646.

9. J. Nielsen, Untersuchen zur Topologie der geschlossenen zweiseitigen Flächen. III, Acta Math. 58 (1932), 87-167.

10. - - Abbildungsklassen endlicher Ordnung, Acta Math. 75 (1942), 23-115. MR 7, 137.

11. H. Rauch, A transcendental view of the space of algebraic Riemann surfaces, Bull. Amer. Math. Soc. 71 (1965), 1-39; Errata: ibid. 74 (1968), 767. MR 35 \#4403; MR 37 \#1591.

12. H. L. Royden, Report on the Teichmüller metric, Proc. Nat. Acad. Sci. U.S.A. 65 (1970), 497-499. MR 41 \#3757.

13. - - Automorphisms and isomorphisms of Teichmüller space, Ann. of Math. Studies, no. 66, Princeton Univ. Press, Princeton, N.J., 1970, pp. 369-383.

14. P. A. Smith, $A$ theorem on fixed points for periodic transformations, Ann. of Math. (2) 35 (1934), 572-578.

Department of Mathematics, Stevens Institute of Technology, Hoboken, NEW JeRSEY 07030 